\title{
Research Papers from the Fifth Edition of the International Conference on lonic Liquid Based Materials (ILMAT-V)
}

\author{
Magdalena Bendova ${ }^{1}$. Johan Jacquemin ${ }^{2}$
}

Published online: 3 March 2022

(c) The Author(s), under exclusive licence to Springer Science+Business Media, LLC, part of Springer Nature 2022

The fifth edition of the International Conference on Ionic Liquid Based Materials (ILMATV) was held from 4 to 8 November 2019, i.e. in those halcyon pre-covid days when we could still travel and socialize freely, in Jussieu University Campus, Paris, France.

This conference series is devoted on the fundamentals and applications of ionic liquids and molten salts. This particular event covered a wide range of topics and fields from electrochemistry, synthesis and catalysis over energy storage to biomolecular applications, photonics and many more.

Responding to the need to create a network of researchers working in this field to enable better collaboration and project consortia creation, the conference also saw the birth of the European Society on Ionic Matter (https://web-esim.eu) that originated from informal and project partnerships of its founding members. This symposium was also strongly supported thanks to the "GDR Liquides Ioniques \& Polymères" sponsored by the French CNRS and by the International Society of Electrochemistry.

The richness of the conference programme was showcased in the plenary and invited talks. For instance, Christian Schroeder (University of Vienna, Austria) talked to us on the computational spectroscopy of ionic liquids. Josefa Fernández (University of Santiago de Compostela, Spain) gave an overview of the tribological utilization of ionic liquid mixtures with nanoparticles. Chris Hardacre (University of Manchester, U.K.) presented a talk on $\mathrm{CO}_{2}$ capture and utilisation in ionic liquids. Last, but not least, Barbara Kirchner (University of Bonn, Germany), who was presented with the Ruth Marion Lynden-Bell award, explored the understanding the molecular cosmos to make ionic liquids better materials. In addition invited speakers demonstrated the great potentials of such ionic solutions for energy storage, biomass dissolution, separation and extraction processes thanks to their fine-tune and unique properties. Such a unique potential was deeply covered during each session thanks to a large panel of oral and flash presentations.

Magdalena Bendova

bendova@icpf.cas.cz

Johan Jacquemin

johan.jacquemin@um6p.ma

1 Department of Chemistry and Physics of Aerosols, Institute of Chemical Process Fundamentals of the CAS, 16502 Prague 6, Czech Republic

2 Materials Science, Energy, and Nano-Engineering MSN Department, Mohammed VI Polytechnic University, Lot 660, Hay Moulay Rachid, 43150 Ben Guerir, Morocco 
The poster session covered similarly broad topics with a focus on electrochemistry and separation processes, the high-quality contributions making it difficult for the scientific committee to select the best poster awardees, which were deeply acknowledged during the Gala Dinner.

Thus, the papers in this Special Issue reflect the scope of the covered subjects and are an interesting crossover between the fundamental and applied research topics presented at the conference. For instance, the paper published by Oriele Palumbo et al. explores the influence of the alkyl chain length on the thermal phase behaviour of imidazolium-based ionic liquids by means a combination of IR spectroscopy and DFT calculations, finding, among others, cation rotational isomerism in one of the ionic liquids studied. Then, Sandrine Bouquillon et al. investigate greener choline-based ionic liquids for dissolution of cellulose and lignin at conditions much milder than those applied conventionally. The article presented by Ana Paula Mora Tavares et al. focuses on the use of aqueous bi-phasic systems that contain polymeric ionic liquids or supported ionic liquid phases in the purification of proteins, discussing the role of the ionic liquid structure and the experimental conditions used in the purification processes. Finally, Ranisha Sharishma Sitlapersad et al. reports a study on the structure and differential capacitance of an electric double layer capacitors by DFT and a comparison between their experimental results with Brownian Dynamics simulations.

Altogether the ILMAT-V conference was a pleasant and stimulating meeting closed by an unforgettable guided tour and dinner at the Musée d'Orsay.

Publisher's Note Springer Nature remains neutral with regard to jurisdictional claims in published maps and institutional affiliations. 